\title{
An Investigation on the Aerodynamic Performance Evaluation and the Layout of Wind Turbine in the Typical Mountainous Terrain
}

\author{
He Hongchuan, ${ }^{1, a}$ and Yuan Wei, ${ }^{1, b}$ \\ ${ }^{1}$ National Key Laboratory of Science and Technology on Aero-Engines Aero- \\ thermodynamics, School of Energy and Power Engineering, Beihang University, Beijing, \\ 100191, China \\ ajiangchuan@buaa.edu.cn, byuanwei@buaa.edu.cn
}

\section{Keywords: Mountainous Terrain; Wind Shear; Wind Power; Layout}

Abstract. The construction of wind turbine generator set depends on the sufficient measurement of wind resources, and reasonable layout and management. Flat open terrain is the ideal condition for the operation of the wind turbine, but the actual terrain fluctuation is changeable. Because these terrains are close to the areas where the electricity is demanded, the wind farm construction and development have more urgent and practical needs. In this paper, a typical mountainous terrain wind shear is studied by numerical simulation. ERS-100 wind turbine is chosen to analyze the performance in the typical mountainous terrain from four respects of wind profile, turbulence intensity, yaw and terrain blockage. The results showed that: turbulence intensity is the important factor affecting the aerodynamic performance, which leads to the large performance loss in nearly $50 \%$ at leeward slope; wind profile near the top of the hill is close to the ideal uniform flow and loss was low, but the performance loss is nearly $30 \%$ at the foot; the installation yaw angle is small, which has a minor effect on performance; terrain blockage only impact on windward slope.

\section{Introduction}

The efficient use of wind energy resources is based on the detailed scientific exploration of wind resources. According to different distribution characteristics of wind resources, the layout of the space of the wind turbine is reasonable, and the state of the wind turbine is monitored and controlled, that is the foundation of the modern wind power plant. Good layout, not only can maximize the benefits of power, but also can guarantee the power grid stability. Different from the flat open terrain, there is wind shear in the near earth space of mountain terrain. The wind shear here refers to the phenomenon that the bottom velocity of the atmosphere is bigger than the upper, which is influenced by the terrain. Wind shear makes the wind speed distribution of mountain terrain no longer consistent with the linear model, so the original experience is not suitable to guide the layout of the wind turbine and the state control.

Due to the complexity of the wind field simulation, the assessments of mountain terrain wind field rely on early experiments. For example, Askervein Hill ${ }^{[1,2]}$ installed the wind tower on the windward slope and leeward slope to explore the characteristics of wind speed. With the development of computational fluid dynamics (CFD) technology and the improvement of the atmospheric wind engineering theory, the numerical simulation of wind resources in the complex terrain of the near earth space is carried out. For the flat terrain, the turbulence characteristics are relative stable and the linear model can meet the evaluation, so the industry generally uses mature commercial software, such as WasP, Park and WindFarmer, etc. ${ }^{[3]}$; for the complex terrain, in addition to the use of the WindSim and the French WT Meteodyn and other software, Fluent and other general computational fluid software for flow field simulationares common technical means.

There are many factors to explore the complex terrain, so the research on the mountain terrain wind field is mainly aimed at the wind speed distribution of the typical mountain. Wei Huirong ${ }^{[4]}$ studied on the distribution of wind velocity in a three dimensional steep slope, and the influence of the roughness length and the slope on the micro-siting of the wind turbine was discussed. Through the typical mountain wind tunnel test and CFD simulation, Guo Wenxing ${ }^{[5]}$ summarized the experience of the CFD simulation. But the performance simulation of the wind turbine in the typical mountainous wind field is still rare. 
The impact of mountainous terrain is a multi-factor for wind turbine. There are different changes in the different positions of the mountain, such as the wind profile, turbulence intensity, the angle of flow and the blockage of the terrain. These factors will affect the performance of the wind turbine. Liao Mingfu et $\mathrm{al}^{[6]}$ pointed out that the effect of wind shear on the performance of wind turbine contains two aspects, wind power loss and machine design power loss. Wu Yongzhong et al ${ }^{[7]}$ used blade element theory by MATLAB method to explore the differences in the performance of a MW wind turbine when the wind profile index is 0.14 . He pointed out that the overall differences in wind turbines and the rotation period different characteristics existed. But the numerical simulation analysis of the wind profile directly on the wind turbine is less. For the aspect of atmospheric turbulence, the turbulence intensity is an important parameter, which represents the velocity fluctuation. Turbulence intensity, in addition to the fatigue damage caused by the structure of the wind turbine, has a serious impact on the efficiency of wind power generation. The greater the turbulence intensity is, the greater the performance $\operatorname{loss}^{[3]}$ is. Through the basic element model method, the influence of turbulence intensity on the performance of the wind turbine is studied by Cao Chong et $\mathrm{al}^{[8]}$, Mountain terrain could change the distribution at the near earth space, so the impact of this change on the performance of wind turbines needs to be evaluated. For the aspect of yaw of the wind turbine, there are stable yaw and instant yaw. Stable yaw, not only comes from the stall adjustment to fit the angle, but also from the mountain terrain effect. In general, there is the $\cos ^{3}$ Yrules between the yaw angle and the power output. Zhao Yinfeng et al ${ }^{[9]}$ verified the applicability of the law to the yaw performance of a small wind turbine. Different wind turbine has different yaw performance, and different installation position has different yaw angle. In addition to the above factors, mountainous terrain changes also can cause blockage of wind turbine wake, resulting in the loss of performance.

In view of the characteristics of the mountain terrain, we need to evaluate the wind shear, turbulence intensity, yaw angle and the influence of terrain on the performance of the wind turbine, so as to obtain the experience of the wind turbine micro layout. In this paper, the typical mountainous terrain wind shear is studied by CFD technology, and the performance of the wind turbine in the typical mountainous terrain is analyzed. When analyzing the causes of the wind turbine performance characteristics at different position, four aspects including the wind profile, turbulence intensity, yaw and terrain will be studied. Finally, the micro layout of the wind turbine will be discussed.

\section{Numerical method}

In the CFD simulation, the neutral stability hypothesis of atmospheric is used in the wind engineering field to ensure that the terrain is the only factor of the wind shear effect. At present, there are some relatively mature conditions for the equilibrium atmosphere, and k- $\square$ turbulence model and k-w turbulence model are mainly based on the field of engineering calculation. The k- $\square$ turbulence model is widely used. Their velocity distributions are similar, which are based on the statistics of the logarithmic law or exponential law; But the turbulent kinetic energy $\mathrm{k}$ and turbulent dissipation rate $\square$ are different, the solution methods for $\mathrm{k}$ and $\square$ can be divided into two categories: Solving the k- $\square$ model equation based on theoretical hypothesis; Based on the experimental data, the assumptive distribution is proposed and then the coefficient can be solved by simultaneous equations. The former representative includes Ferziger and Peric ${ }^{[10]}$, Richard and Hoxey ${ }^{[11]}$ et al. The latter representative includes Yang $\mathrm{Yi}^{[12]}$ et al. Though the latter is better when comparing compliance between CFD and experiment, and the self-preserving of parameters is also higher, but the fitting parameters of the coefficients are too dependent on the experimental data to be universal. So the method of Ferziger and Peric is chosen ${ }^{[5,10]}$ in this study.

ANSYS Fluent software is used to analyze the flow field. RNG k- $\square$ turbulence model is chosen for use ${ }^{[5]}$. Turbulence boundary conditions are input through the user defined function. Equations solving uses the Couple algorithm. The momentum equations and the model equations are discretized by using the second order upwind scheme. The blade profiling of wind turbine uses the 
SIEMENS UG software, and the flow simulation model is based on a multi-rotation reference frame.

\section{Wind shear near earth space}

Good retention of the wind profile is the basis for the research on the influence of the construction and the terrain on the wind speed in the near earth space. Generally, the distribution of wind velocity in the atmospheric turbulence is expressed by the wind profile, which follows the rules of the logarithm law or index law. Firstly, self-preserving of the velocity profile in the near earth space is verified, and then the wind field parameters in typical mountainous terrain will be analyzed as a comparison.

Neutral stability hypothesis of atmospheric near earth. The neutral stability hypothesis of atmospheric near earth means the self-preserving of the velocity profile in the near earth space, and this hypothesis requires reasonable atmospheric turbulence flow conditions. The wind profile index $\alpha$ and roughness length $y_{0}$ are important parameters. In order to unify them near earth, we can refer to the relationship ${ }^{[13]}$ :

$$
\alpha=\alpha_{0}\left[1-0.55 \lg \bar{V}\left(y_{\text {ref }}\right)\right], \alpha_{0}=\left(\frac{y_{0}}{10}\right)^{0.2}
$$

In present study, turbulence intensity refers to the $\mathrm{AIJ}^{[14]}$. For the selected wind speed profile index, the turbulence intensity in the $\mathrm{y}_{\mathrm{b}}$ height is conducted smooth handling, and the correction value is also within the standard. Because of the symmetry, 2-D numerical model is usually used to verify the self-preserving of the atmospheric boundary layer. The flow field $(1000 \mathrm{~m} \times 500 \mathrm{~m})$ is set up for the model. In order to establish the coordinate system, the wall center is selected as the origin of coordinates, and the flow direction is X axis. The structured grid is used as shown in Fig. 1, and the boundary conditions are shown in Tab. 1

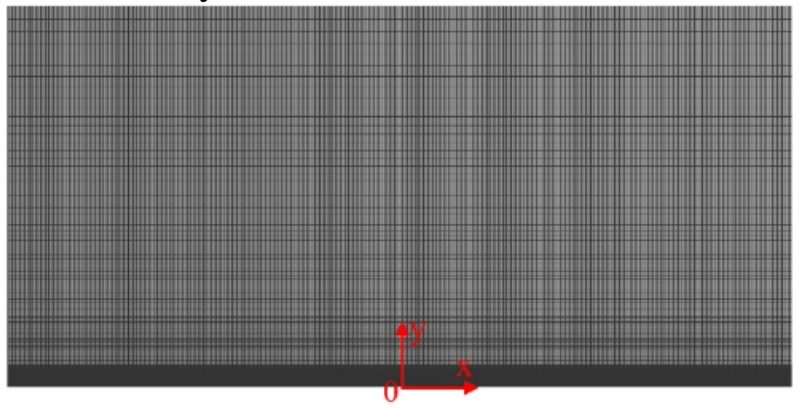

Fig. 1 2-D flow field mesh
Tab. 1 Boundary conditions

\begin{tabular}{|c|c|}
\hline $\begin{array}{l}\text { Turbulence } \\
\text { Boundary } \\
\text { Condition }\end{array}$ & $\begin{aligned} U=U_{\text {ref }} \times\left(\frac{y}{y_{r e f}}\right)^{\alpha}, k=\frac{3}{4} \times(u \cdot I)^{2}, \varepsilon=C_{\mu}^{\frac{3}{4}} \times \frac{k^{\frac{3}{2}}}{K y} \\
I=\left\{\begin{array}{l}0.222, y \leq y_{b} \\
0.1 \times\left(\frac{y}{y_{G}}\right)^{-\alpha-0.05}, y_{b}<y \leq y_{G} \\
0.1, y>y_{G}\end{array}\right.\end{aligned}$ \\
\hline $\begin{array}{c}\text { Wall } \\
\text { Function }\end{array}$ & $k_{s}=y_{0}, C_{s}=0.5$ \\
\hline
\end{tabular}

When the residual error is below $1 \times 10^{-10}$, the convergence is achieved. The wind profiles and turbulence intensity at three reference sections including "X=0", inlet and outlet, will be compared. As shown in Fig. 2(a), the boundary conditions can be self-preserving at $500 \mathrm{~m}$ height of the wind profile. And in $50 \mathrm{~m}$ height near earth where the wind turbine locates, the maximum speed change is no more than 3\%, as shown in Fig. 2(b).

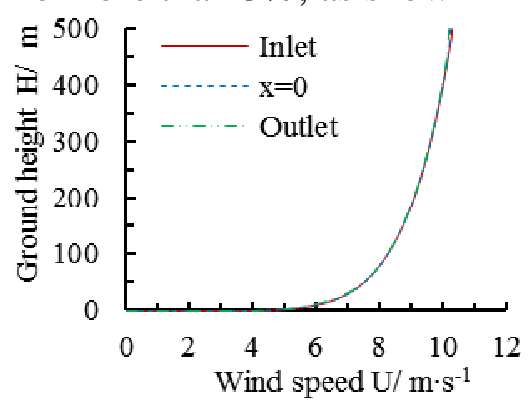

(a)

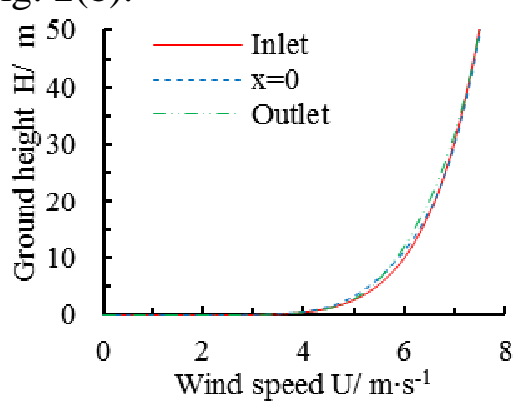

(b)

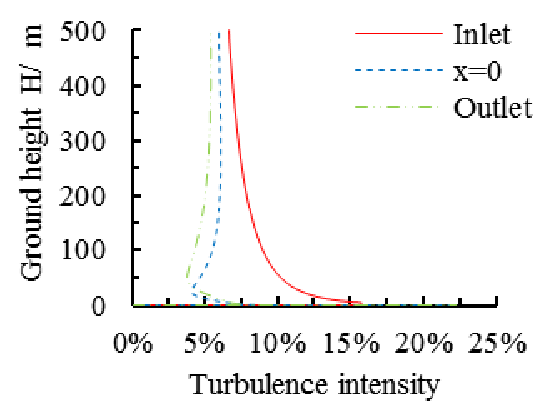

Fig. 3 Variation of Turbulence intensity distribution

From Fig. 3, we can see that the change of the turbulence intensity is more obvious than velocity, and the overall has a depressed trend, but the distribution law can meet the requirements of wind engineering. In summary, self-preserving properties have been verified. The boundary conditions can explore the wind shear.

Typical mountain terrain wind shear. The Gaussian model is used as the description of the 
mountain. The equation is as follows:

$$
y=h \cdot e^{-0.5\left(\frac{x}{\sigma}\right)^{2}}, \sigma=\frac{l}{1.1774}
$$

It is generally accepted that too large slope of the mountain will lead to the existence of separation. So a large slope is not particularly significant for the exploration of the wind shear in mountainous terrain. In present study, As shown in Fig. 4, the main parameter is $l=2 h$, where " $l$ " is the axis distance corresponding to half mountain height.

Because the slope is not steep, it will not cause separation. 2-D flow field is also used. The flow direction is $\mathrm{X}$ axis, and the $\mathrm{X}$ axis position of the top of the mountain is defined as the origin of coordinates. Grid is shown in Fig. 5. Wind profile and turbulence intensity at $x=0, \pm 50, \pm 100, \pm 150, \pm$ $200, \pm 300 \mathrm{~m}$ will be discussed.

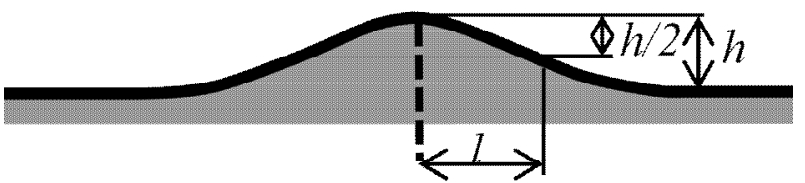

Fig. 4 Schematic diagram of mountain

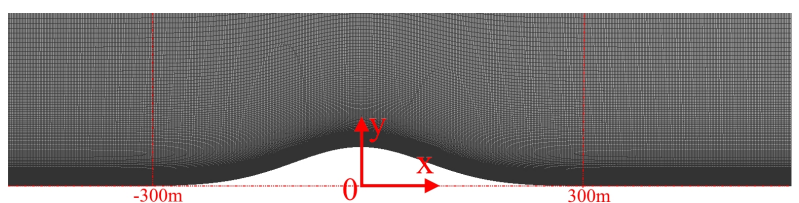

Fig. 5 2-D mountain flow field mesh

As shown in Fig. 6(a), the wind profile at $\pm 300 \mathrm{~m}$ has little change. The wind profiles become more and more full toward the top of the mountain, and there is an obvious accelerated wind shear on the top of the mountain. On both sides of the mountain, there is a similar wind profile from the ground. But there is a big difference in the near earth space, as shown in Fig. 6(b).

The changes in the terrain are also reflected in the turbulence intensity of space. As shown in Fig. 7(a), in the upper space, the terrain has a very small impact. But as shown in Fig. 7(b), The turbulence intensity dramatically changes in the leeward slope position near the earth space, especially in $20 \mathrm{~m}$, where is adverse to the wind turbine.

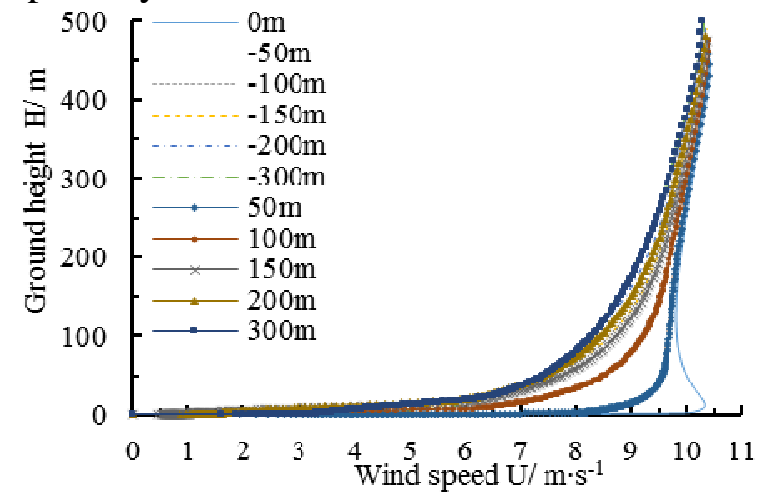

(a)

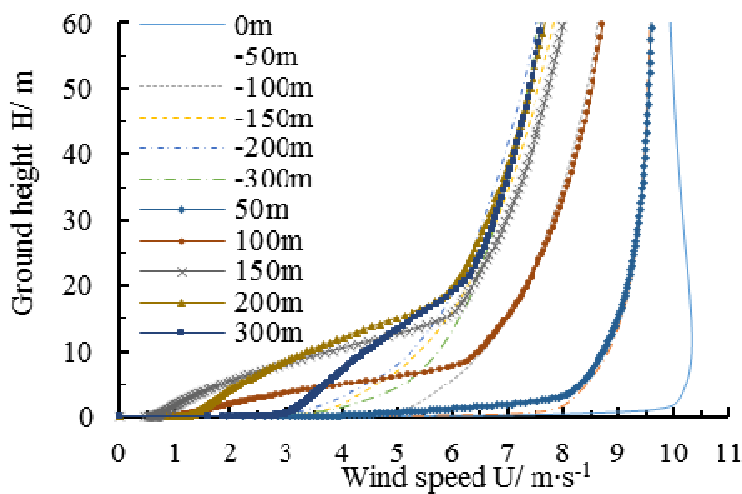

(b)

Fig. 6 Variation of wind profile along the terrain

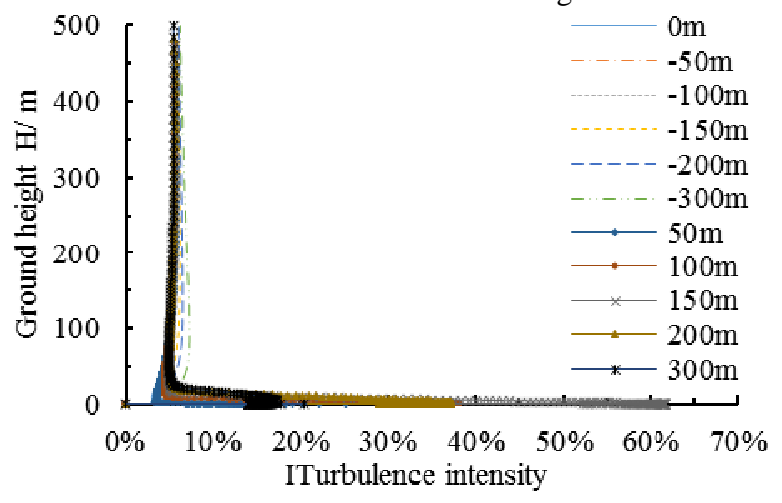

(a)

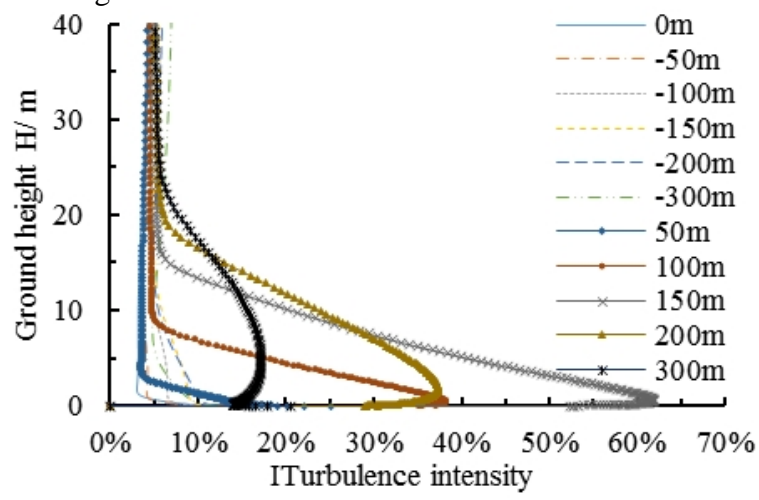

(b)

Fig. 7 Turbulence intensity changes along the terrain

The wind speed distribution along slope of the mountain is compared in different tower height. Parameters have been non-dimensionalized. As shown in Fig. $8(0.1 \mathrm{~h}$ represents $5 \mathrm{~m})$, the incoming wind speed $\mathrm{U}_{0}$ is the speed dimension parameter, and the height of the mountain $\mathrm{H}$ is the length dimension parameter. 
When the tower height from the ground is lower, the acceleration effect of the terrain is more obvious, and the recovery of the downstream wind speed is slower, so the mountain terrain impacts farther away. But near the ground of the bottom of the mountain, the wind speed decreases due to the blockage in the upwind slope, while the wind speed of leeward slope decreases due to the basin expansion. At $10 \mathrm{~m}$ height, the deceleration near the bottom of the mountain in the upwind side can reach $20 \%$, and near the bottom of the mountain in the Leeward side can reach $40 \%$. The peak acceleration ratio can reach $75 \%$ at the top of the mountain. This conclusion is basically the same with Askervein Hill ${ }^{[1,2]}$.

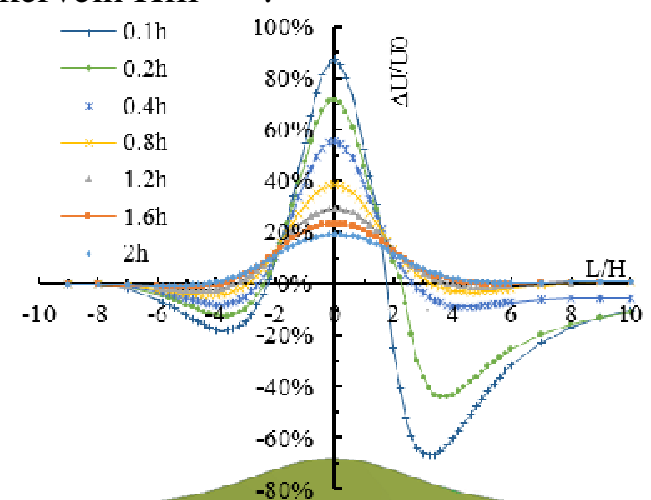

Fig. 8 Acceleration deceleration ratio of different ground height

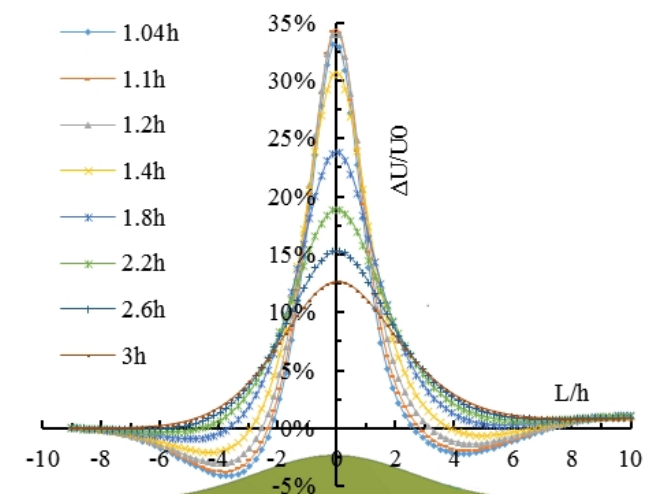

Fig. 9 Acceleration and deceleration ratio of different absolute height

The increase in wind speed, on the one hand, is due to the acceleration effect of the mountain. On the other hand, is also due to the absolute height from the sea level. The wind speed at different absolute height from the sea level will be compared. As shown in Fig. 9 (1.0h represents $50 \mathrm{~m}$ ). The lower the height is, the greater the influence of the terrain is. The maximum acceleration ratio reaches 34\%; and with the increase in absolute height, the acceleration and deceleration effect caused by the mountain reduces. When the absolute height reaches $150 \mathrm{~m}$, the mountain terrain effect is cut down below $12 \%$.

\section{Performance in typical mountain}

The wind turbine works well in the steady wind condition, but the actual terrain is complex. According to experience, the severer the actual working condition is, the worse the performance loss is. In this part, ERS-100 is chosen to simulate the performance of wind turbine on mountain terrain. Airfoil of ERS-100 is based on the high lift airfoil (S821, S819 and S820) of the NEREL ${ }^{[15]}$. Its rated performance ${ }^{[16]}$ is shown in Fig. 10.

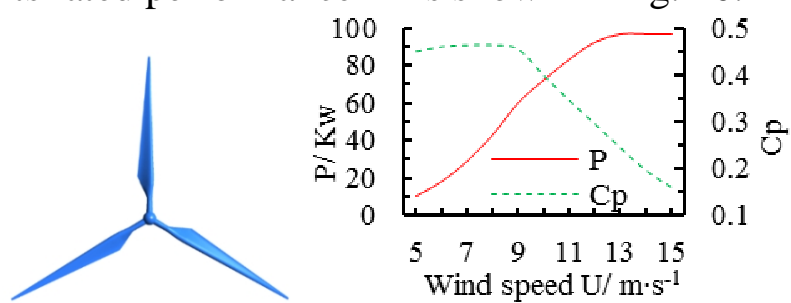

Fig. 10 Model and Aerodynamic performance of ERS-100



Fig. 11 Model and mesh of typical mountainous terrain

Wind turbine Performance simulation. The mountain model is the same as before. The computational model is set for a 3-D steady incompressible flow fields (1000 m×200 m $\times 500 \mathrm{~m})$, as shown in Fig. 11, and the coordinates are as same as Fig. 5. $U_{\text {ref }}$ is set to $8 \mathrm{~m} / \mathrm{s}$ in this part and other parameters adjusts correspondingly. The effect of tower and tilt of the impeller are ignored, and only the impact of mountain terrain on the wind turbine performance is assessed. The number of grid is 1800000, as shown in Fig. 11.

As shown in Fig. 12, the wind speeds are different along different positions. Following the control rules of ERS-100, the maximum power can be gained by adjusting the rotating speed and raw angle. Because of the wind shear on the mountain terrain, the actual power is not cube of wind speed.

In order to evaluate the influence of terrain on the wind turbine, the capacity factor is introduced: 


$$
F=\frac{P_{\text {cal }}(U)}{P_{\text {rated }}(U)}
$$

Where $F$ represents the influence of terrain on wind energy conversion. As shown in Fig. 13, the capacity factor varies greatly along the mountain, the capacity factor on the leeward slope of $100 \mathrm{~m}$ is only $53 \%$, and only $66 \%$ near the foot.

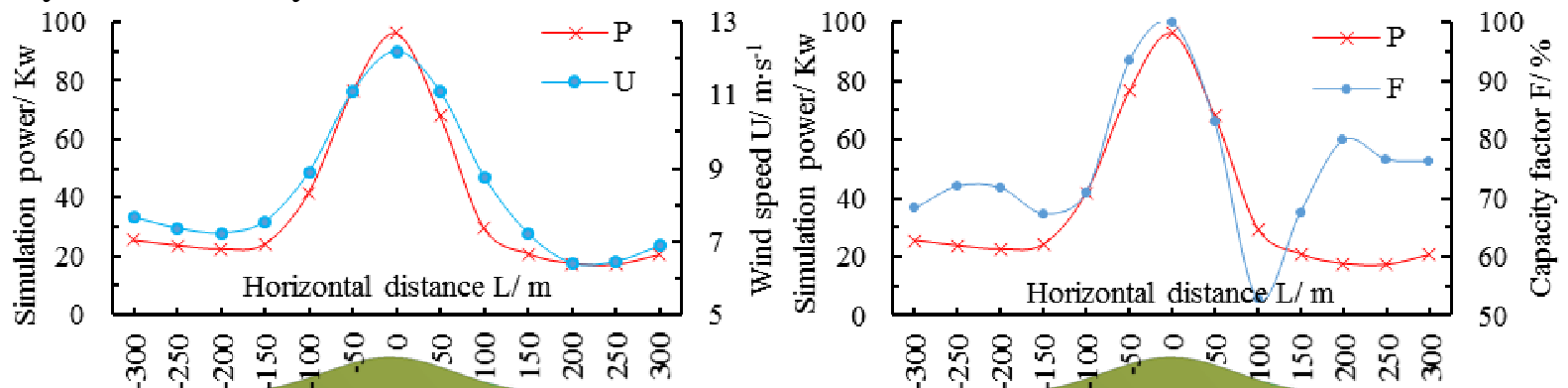

Fig. 12 Distribution of wind speed and Simulation power

Fig. 13 Distribution of simulation power and capacity factor

For the performance of the ERS-100 wind turbine, there is some difference between the model calculation and the rated value. Most of the hillside performance has not reached the expected because of the impact of mountain terrain.

For a wind turbine working in mountainous terrain, the working conditions mainly have three characteristics: yaw, wind shear and turbulence intensity. Of course, the blockage effect cannot be ignored because the terrain changes. Because of the difference in the mountain and the size and performance of the wind turbine, there are different effects of response. In order to separate the influence factors, the performance of the wind turbine is analyzed in the single influence factor. Here a fast simulation model is set up. The number of grid is 1400000. As shown in Fig. 14, the effects of turbulence intensity and yaw angle are analyzed.

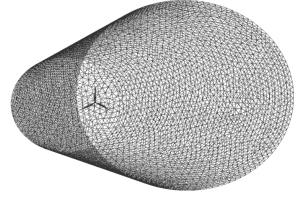

Fig. 14 ideal state mesh of ERS-100

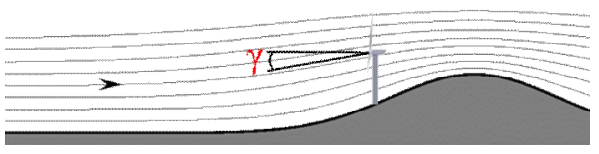

Fig. 15 Schematic diagram of Yaw influence on the mountains

Effect of turbulence intensity. The pre-set turbulence intensity of the inlet flow is $5 \%, 20 \%$ and $40 \%$, respectively in the range of ERS-100 operating speed. The relationship between wind speed and power under different turbulence intensity will be obtained, and the performance on the mountain terrain will be compared with the rated value, as shown in Fig. 16.

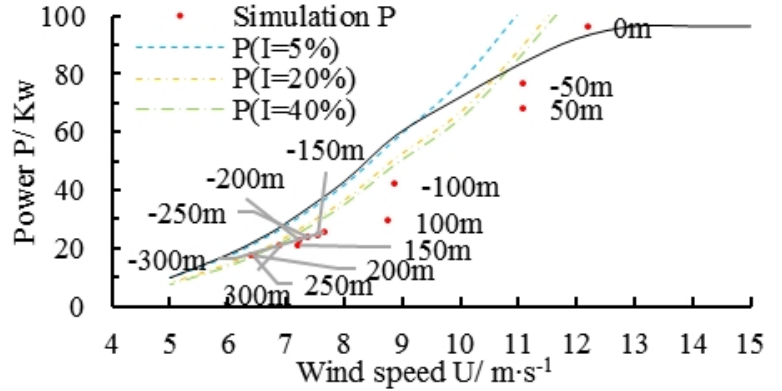

Fig. 16 Effect of turbulence intensity on ERS-100 performance

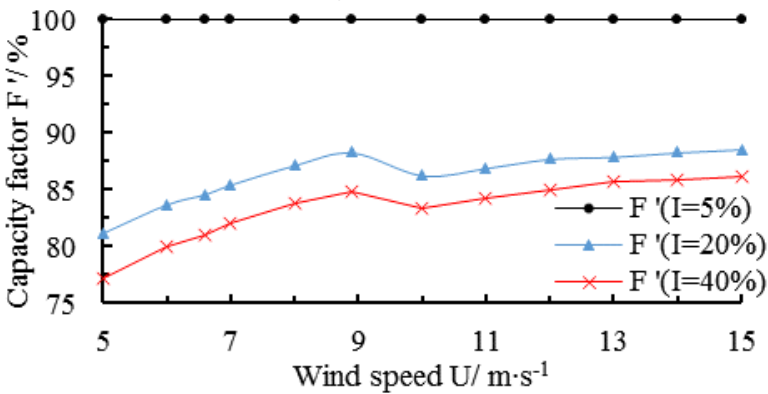

Fig. 17 Effect of turbulence intensity on the capacity factor

From Fig. 16, it is shown that when the turbulence intensity increases gradually, the power $\mathrm{P}$ is reduced, which is coincident with the practical engineering experience. When the turbulence intensity is $20 \%$, which is close to the standard turbulence intensity $23 \%$ in wind field, the power of the wind wheel is reduced by about $17 \%$. At the same time, it can be found that the error caused by the calculation is increasing, mainly due to the turbulence model. The calculation beyond the optimized TSR is not accurate. In order to obtain the influence of the turbulence intensity on the performance of the wind turbine, the low turbulence intensity is set to $5 \%$ as a metewand for capacity factor F. As shown in Fig. 17, the inflection point of the $9 \mathrm{~m} / \mathrm{s}$ in the picture is just the design of the TSR, which can't be maintained by the speed of rotation.

As can be seen from Fig. 7(b), turbulence intensity of the leeward slope increased in the near 
earth space from $10 \%$ up to $40-50 \%$, which leads to the operating points far away from the design state at $50 \mathrm{~m}$ and $100 \mathrm{~m}$ on leeward slope. The range of the turbulence intensity changes between $10 \%$ and $20 \%$ on the windward slope. While the variations range of turbulence intensity is $20 \% \sim 60 \%$ on the leeward slope. So the leeward slope has great influence on turbulence intensity.

Effect of yaw angle. From the previous studies, the larger the diameter of the wind turbine is, the smaller the variation of the ground surface caused by the terrain is. The ERS-100 with diameter of $20 \mathrm{~m}$ and height of $50 \mathrm{~m}$ will have an effect when it is installed on mountain terrain. The wind turbine cannot be orthogonal to the ground on the mountain. So there is a yaw angle when the wind turbine is rotating, as shown in Fig. 15.

Firstly, the research on the yaw performance of ERS-100 is studied. Under the absolute reference system, the simulation of the yaw angle is composed by the axial velocity component and the normal velocity component. The flow velocity is $8.9 \mathrm{~m} / \mathrm{s}$. The relationship between the capacity factor and the yaw angle is obtained under the yaw angle $0^{\circ} \sim 40^{\circ}$, At the same time, as shown in Fig. 18, the yaw performance of ERS-100 follows the $\cos ^{3}$ Yrules.

The angle between the wind speed at the height of the tower and the axial direction of the impeller is defined as the yaw angle. The relationship between position and yaw angle is established, and also the relationship between the capacity factor and the yaw angle is established by applying the $\cos ^{3}$ Yrules. As shown in Fig. 19.
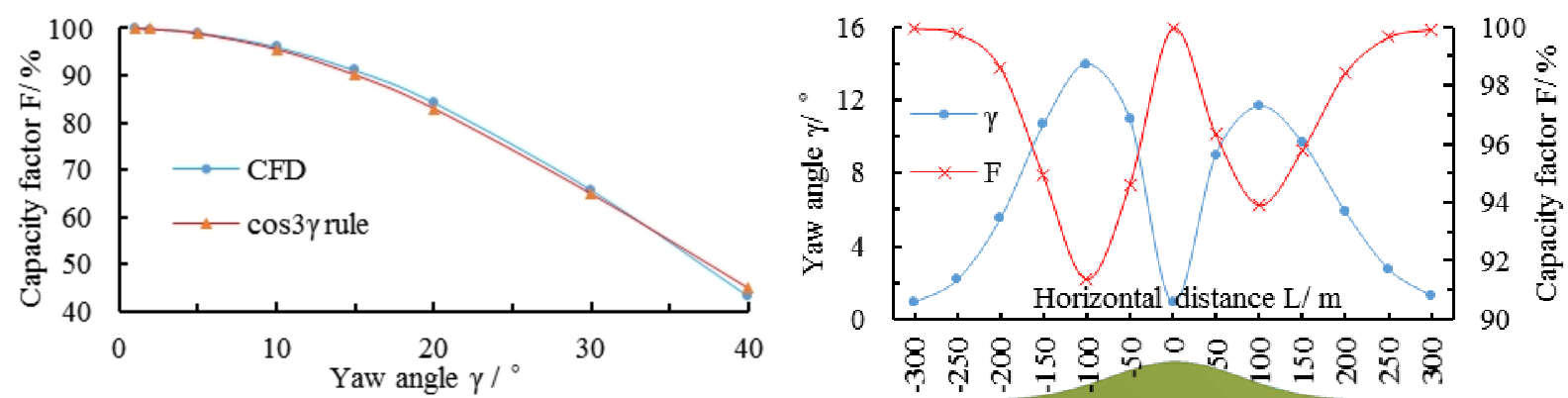

Fig. 18 Relationship between yaw angle and capacity factor

Fig. 19 Distribution of the yaw angle and its capacity factor along the mountain

The yaw angle of hillside is the largest. The upwind yaw angle reached $14^{\circ}$. The downwind yaw angle reached $12^{\circ}$. The corresponding performance decreases by $9 \%$ and $6 \%$. At the foot of the mountain and the top of the hill, the yaw angle is close to $0^{\circ}$, and the effect can be neglected, and the performance changes at the other position are less than 5\%. Of course, the smaller the diameter of the wind wheel is, the more obvious the effect will be.

Effect of wind profile and blockage. The ideal incoming flow for the wind turbine is uniform, but the actual incoming flow conditions are complicated. In mountainous terrain, the wind profile becomes fuller and fuller when the location closes to the top of the mountain, and the flow field turns to be uniform, so the performance is more close to the rated power. Near the foot of the mountain, the wind profile is similar with the atmosphere boundary layer(ABL) profile, as shown in Fig. 6. In order to separate the influence of the wind profile and the mountain block, a model of flat terrain flow field is set up, and a comparison of performance between the flat terrain and the foot of the mountain will be conducted. As shown in Fig. 20.

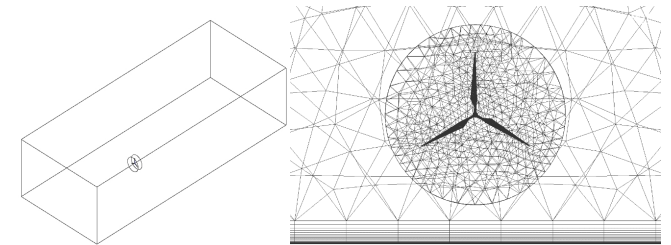

\begin{tabular}{cccc} 
Tab. 2 Different performances of similar wind profiles \\
& Wind speed $[\mathrm{m} / \mathrm{s}]$ & $\mathrm{P}[\mathrm{Kw}]$ & $\mathrm{F}$ \\
\hline ABL & 7.8 & 28 & $72 \%$ \\
Upwind foot & 7.66 & 25 & $66 \%$ \\
Leeward foot & 6.88 & 20 & $73 \%$ \\
\hline
\end{tabular}

Fig. 20 Flow field of wind turbine on flat terrain

As shown in Tab. 2, at the foot of the hill, the performance is less than that on flat terrain by $6 \%$. This difference comes from the blockage effect generated by the terrain.

Based on the combination of turbulence intensity and yaw angle, such results can be drawn: At the foot of the mountain $(-300 \mathrm{~m} \sim-200 \mathrm{~m})$, the influence of the yaw angle on the performance is small, and the influence of the turbulence intensity represents a quite fraction, the rest is the result of the 
combined effects of wind profile and terrain blockage.

In order to evaluate the relationship between the different factors affecting the performance of the wind turbine, the relationship between the total capacity factor (the 5\% turbulence intensity performance is the metewand) and the coefficient of each factor is established:

$$
F^{\prime}=F_{Y}^{\prime} \cdot F_{I}^{\prime} \cdot F_{W}^{\prime} \cdot F_{B}^{\prime}
$$

Here: $Y$ represents yaw, $I$ represents turbulence intensity, $W$ represents wind profile, $B$ represents blockage. The influence of each factor is:

$$
\eta_{i}=\frac{\ln F_{i}^{\prime}}{\ln F^{\prime}} \times 100 \%
$$

Under the condition of the atmosphere, the capacity factor of the yaw angle is $100 \%$, the capacity factor of the turbulence intensity is $90 \%$, and the capacity of the wind profile is $80 \%$. Combined with the performance at the foot of mountain under the similar flow condition, the capacity factor of the block is about $92 \%$. In the same way, the capacity factor of other factors affecting the wind turbine along the different position of the mountain can be obtained.

In order to analyze the performance loss on different positions of the mountain, the loss coefficient is defined as following:

$Q=1-F$

The influence of different factors on $Q^{\prime}$ (the metewand as same as $F^{\prime}$ ) is:

$$
Q_{i}^{\prime}=Q^{\prime} \cdot \eta_{i}
$$

It is not difficult to find the impact of different factors on the loss coefficient, as shown in Fig. 21.

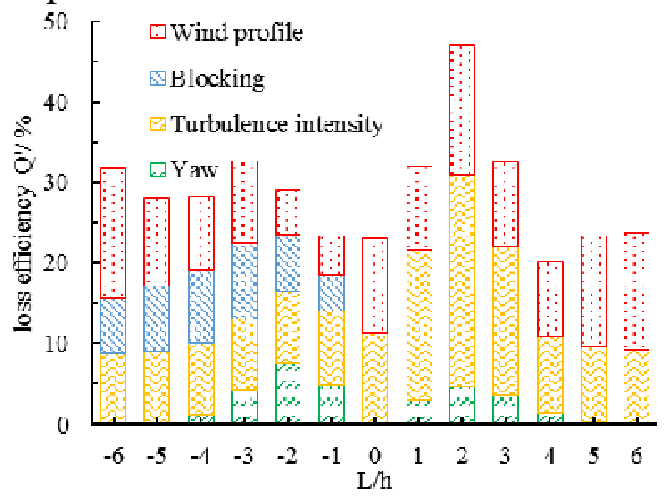

Fig. 21 Proportion of losses caused by various factors

Leeward slope loss mainly comes from the increase of turbulence intensity and wind velocity attenuation near earth, and the loss is serious. In the upwind slope, the loss caused by the blocking performance and the effect of the yaw is less. The wind profile near the top of the mountain is the closest to the uniform flow, and the impact is the minimum. Overall: Near the top of the mountain, the performance loss of the wind turbine is the minimum, and the power output increases with the increase of the wind speed; The performance on the windward slope is better than that on the leeward slope, but the foot of the mountain is not a good choice. Although loss coefficient on leeward slope is not high, it is due to the decrease in wind speed, and may not be suitable for the arrangement of the wind turbine. These are in accordance with the actual experience of engineering.

\section{Conclusions}

a. The typical mountain terrain will increase the wind speed, resulting in the speed change on windward and leeward slope is nearly symmetrical. With the decrease of the height on the Lee slope, the wind speed loss is more pronounced, and the turbulence intensity increases significantly, which can reach the maximum value near to the halfway up the mountain. The mountain height and the size of wind turbine determine the scope of influence. The smaller the diameter of the wind wheel is, the more obvious the impact of the mountain has;

b. When the wind turbine is arranged on the hillside, the yaw angle, the turbulence intensity, the wind profile and the mountain block will have different effects in different positions. Using capacity factor, the influence factors of different position are compared: Yaw angle has less impact 
on the performance, and the yaw angle reached the maximum on the halfway of the mountain; The turbulence intensity increases is a major factor in the leeward slope performance loss; The influence of the wind profile on the performance is obvious, whether it is flat or mountainous; The wind profile is the fullest on the top of the mountain, and the performance is the best; The blockage of the mountain mainly happens on the upwind slope, the most obvious influence is near the foot of the mountain. Overall, the wind turbine layout on the windward slope is reasonable, and layout on the top of the hill is better than that at the foot, and the biggest loss happens on the halfway of the leeward slope;

c. The layout on the typical mountain terrain in the form of a single wind turbine is regular. But the wind farm layout is more complex. In addition to the more complex wind shear, the layout of the wind turbine will also affect the installed capacity: Although the wind turbine near the top of mountain can bring the maximum power, its wake will influence the downstream wind turbine layout and the installed density, so layout for the wind farm needs to further explore to realize the optimization.

\section{Acknowledgement}

This investigation was supported by the Fundamental Research Funds for the Central Universities (Grant No. YWF-15-GJSYS-018).

\section{References}

[1] Taylor P A, Teunissen H W. Boundary-Layer Meteorology. 1987, 39(1-2): 15-39.

[2] Mickle R E, Cook N J, Hoff A M, et al. Boundary-Layer Meteorology. 1988, 43(1-2): 143-169.

[3] Li Z, He D. Advances in Mechanics. 2013(05): 472-525.

[4] Wei R. The numerical simulation of windfarm micro-siting. North China Electric Power University, 2007.

[5] Guo W. CFD multi-scale simulation of wind flow over hilly terrain. Harbin Institute of Technology, 2010.

[6] Liao M, Xu K, Wu B, et al. Journal of Shenyang University of Technology. 2008(02): 163-167.

[7] Wu Y, Wang H. Energy Engineering. 2011(06): 33-35.

[8] Cao C, Mao S, Zhou X. Guangdong Electric Power. 2011(05): 29-33.

[9] Zhao Y, Liu Z, Guo Y. Power \& Energy. 2011(05): 395-396.

[10] Ferziger J H, Peric M. Computational methods for fluid dynamics. Springer Science \& Business Media, 2012.

[11] Richards P J, Hoxey R P. Journal of Wind Engineering \& Industrial Aerodynamics. 1993(93): 145-153.

[12] Yang Y, Gu M, Chen S, et al. Journal of Wind Engineering and Industrial Aerodynamics. 2009, 97(2): 88-95.

[13] Spera D A, Richards T R. NASA STI/Recon Technical Report N. 1979, 80: 13623.

[14] AIJ. Recommendations for Loads on Buildings. Japan: 2006.

[15] Somers D M. The S819, S820, and S821 Airfoils. Airfoils, Inc. 1993.

[16] Berry D. Design of 9-meter carbon-fiberglass prototype blades. Technical Report. 2007. 